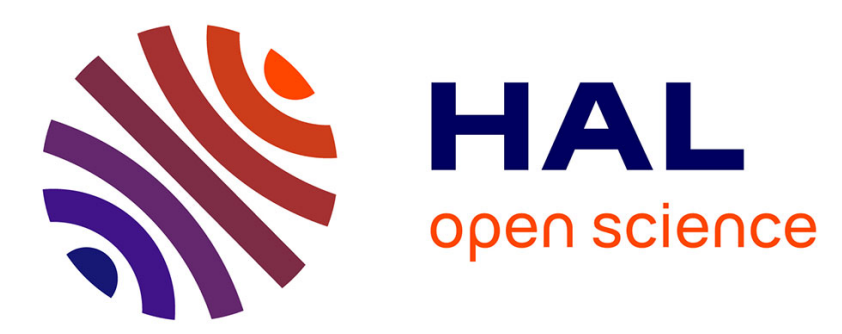

\title{
An Iterative Linear DistFLow for Dynamic Optimization in Distributed Generation Planning Studies
}

Remy Rigo-Mariani, Vannak Vai

\section{To cite this version:}

Remy Rigo-Mariani, Vannak Vai. An Iterative Linear DistFLow for Dynamic Optimization in Distributed Generation Planning Studies. International Journal of Electrical Power \& Energy Systems, 2022, 138, pp.107936. 10.1016/j.ijepes.2021.107936 . hal-03528444

\section{HAL Id: hal-03528444 \\ https://hal.science/hal-03528444}

Submitted on 17 Jan 2022

HAL is a multi-disciplinary open access archive for the deposit and dissemination of scientific research documents, whether they are published or not. The documents may come from teaching and research institutions in France or abroad, or from public or private research centers.
L'archive ouverte pluridisciplinaire HAL, est destinée au dépôt et à la diffusion de documents scientifiques de niveau recherche, publiés ou non, émanant des établissements d'enseignement et de recherche français ou étrangers, des laboratoires publics ou privés. 


\title{
An Iterative Linear DistFLow for Dynamic Optimization in Distributed Generation Planning Studies
}

\author{
Rémy Rigo-Mariani ${ }^{1 *}$, Vannak Vai ${ }^{2}$ \\ ${ }^{1}$ Univ. Grenoble Alpes, CNRS, Grenoble INP, G2Elab, 23 rue des Martyrs, 38000 Grenoble, France \\ ${ }^{2}$ Department of Electrical and Energy Engineering Institute of Technology of Cambodia (ITC) PO Box 86, \\ Russian Confederation Blvd, Phnom Penh, Cambodia \\ *remy.rigo-mariani@g2elab.grenoble-inp
}

\begin{abstract}
This paper implements an iterative linear DistFlow for the modelling of radial distribution grids. The method is intended for dynamic optimizations under gird constraints, and in the presence of distributed generation including storage units with time coupled constraints. It is demonstrated that traditional piecewise linearization for the losses estimation in conventional linear DistFlow can lead to significant errors. This is due in particular to the setting of static upper bounds for the active and reactive branch flows in the linearization process, which may differ greatly from the actual power. The proposed iterative approach addresses this shortcoming with successive runs of linear DistFlow and updates for the flows upper bounds, dynamically along the simulated horizon. The method is compared to conventional linear DistFlow as well as other relaxed formulations such as Second Order Conic Programming and Quadratic Programming. All the methods are discriminated with regard to a reference AC power flow, in terms of error for the voltages and losses profiles on different test systems. The proposed iterative procedure displays the lowest error for the line losses with five to forty times more accuracy than conventional linearized formulations. It also outperforms the Second Order Conic relaxation in terms of scalability with one month dynamic simulation (at $1 \mathrm{~h}$ time step) run in 7 min with 30 distributed units on a 69-bus system. The approach is further validated with typical uses cases for the operation, the sizing, and the siting of distributed assets consisting of solar generators and storage units. Especially, the procedure is coupled with a genetic algorithm in order to test different system configurations on a 90-bus system. The solutions are discriminated in terms of number of assets, installed capacities, connection bus(es), installation costs, system losses and system self-sufficiency.
\end{abstract}

1. Nomenclature.

Sets :

$t \in T$

$b \in B$

$l \in L$

$c \in C$

$b \in D_{b}(l) \subset B$

$l \in D_{l}(l) \subset L$

$s(l), e(l)$

set of time steps

set of buses ( $B$ buses)

set of branches ( $L$ lines)

set of piecewise segments ( $K$ segments)

set of all the buses downstream a line $l$

set of all the lines downstream a line $l$

start/end buses of line $l$

Operation Variables :

$p_{l, t}, q_{l, t} \quad$ active and reactive powers in line $l$ at time $t$

$\delta p_{l, t}, \delta q_{l, t}$ active and reactive powers in line $l$ at time $t$

$I_{b, t}$ square current in line $l$ at time $t$

$v_{b, t}, V_{b, t}$ voltage and square voltage at bus $b$ at time $t$

$p_{l, k, t}^{+}, p_{l, k, t}^{-}$ line $l$ active (+/-) power in block $k$ at time $t$

$q_{l, k, t}^{+}, q_{l, k, t}^{-}$ line $l$ reactive (+/-) power in block $k$ at time $t$

$P_{b, t}^{s t+}, P_{b, t}^{s t-}$ charge/discharge of battery at bus $b$ at time $t$

$S O C_{b, t}^{\mathrm{st}}$ state of charge of storage at bus $b$ at time $t$

$P_{b, t}^{p v}$ solar generation at bus $\mathrm{b}$ at time $t$

Sizing Variables - (parameters for siting only)

$P_{b}^{p v R} \quad$ rated solar generation at bus $b(\mathrm{kWp})$

$P_{b}^{s t R} \quad$ rated power of storage at bus $b(\mathrm{~kW})$

$E_{b}^{s t R} \quad$ rated capacity of storage at bus $b(\mathrm{kWh})$

Parameters :

$P_{b, t}^{L}, Q_{b, t}^{L} \quad$ active and reactive load at bus $b$ at time $t$

\begin{tabular}{|c|c|}
\hline $\begin{array}{l}\overline{p_{l, t}}, \overline{q_{l, t}} \\
v_{b}, \overline{v_{b}}, \overline{i_{l}}\end{array}$ & $\begin{array}{l}\text { line } l \text { active reactive power upper bound at time } t \\
\text { bus } b \text { voltage limits and line } l \text { current limit }\end{array}$ \\
\hline$x$ & resistance and reactance of line $l$ \\
\hline$\eta_{b}^{\text {bat }}$ & battery efficiency at bus $b$ \\
\hline$P_{t}^{p v N}$ & normalized solar generation at time $t$ \\
\hline$C^{P p v R}$ & cost of solar installation $(\$ / \mathrm{kWp})$ \\
\hline$C^{P s t R}, C^{E s t R}$ & cost of battery installation $(\$ / \mathrm{kW}, \$ / \mathrm{kWh})$ \\
\hline$\alpha^{s s r}$ & system self-sufficiency ratio \\
\hline$v_{0}$ & reference voltage (= 1 p.u.) \\
\hline \multicolumn{2}{|c|}{ Iteratively Updated Parameters } \\
\hline$\overline{p_{l, t}^{k}} \overline{q_{l, t}^{k}}$ & Upper bound flow in line $l$, time $t$ and iteration $k$ \\
\hline$V_{b, t}^{k}$ & square voltage at bus $b$, time $t$ at iteration $k$ \\
\hline.$* k$ & upperscript for the optimal results at iteration $k$ \\
\hline
\end{tabular}

2. Introduction

The benefits of distributed generation (DG) integrated into conventional power systems have long been identified with losses and emissions reduction, improved voltage profiles and system reliability, as well as the opportunity to defer significant grid investments [1]. DG planning then refers to the optimal allocation of the resources in terms of type, size and site [2], which have been extensively addressed in the literature for the past two decades. One main technical challenge is that the considered systems have to be simulated a great number of times, and with different DG configurations before finding the best solution [3], which may lead to 
prohibitive computational times. Especially, solving the nonlinear load flow equations over long time horizons may result in non-tractable problems as pointed out in [4] and [5]. In order to overcome that challenge, most of the solutions encountered in the literature (over $80 \%$ of the reviewed papers in [6]) do not consider time-varying load, but limited numbers of load levels ('snapshot mode' as in [7], [8] or [9] as opposition to dynamic optimization). The optimal power flow (OPF) is then run independently for a small set of load values with 'multi periods' successive power flow using the full AC equations [10] that can be solved using metaheuristics such as differential evolutionary as in [11] or genetic algorithm as in [12]. However, the optimality cannot be guaranteed and the convergence may not be ensured for large scale problems with those methods. Convex relaxations such as Second Order Conic Programming (SOCP) [13] are oftentimes favoured, and computational times reduced with limited number of load levels considered in many cases That is justified by the implemented planning stratgies, which often consist in integrating the load flow analysis in metaheuristics (e.g. particle swarm optimization, genetic algorithm). Those algorithms generate great numbers of different DG configurations to evaluate ([10] [7]) and cannot afford long computation times for every tested scenario. However, such 'snapshot' or 'multi-periods' OPF can no longer be considered if DG resources include storage units. In such cases, time coupled constraints shall be embedded in dynamic optimization and the OPF is then integrated in the simulation of the system over a representative period (day, week, month, year) [5]. One way to leverage that complexity is to consider DC approximation for the flow equation [14] at the cost of extensive simplifications that are not relevant for planning problems in distribution networks. Simpler DistFLow equations for radial systems [15], and especially their quadratic (QDF) [16] or linearized (LDF) equivalent [17], are a good compromise which allows to run simulations over longer time horizons and are oftentimes used for distributed control strategies [18] or planning problems [19]. Typical linear DistFlow formulations estimate the line losses with a piecewise linearization (PWL) of the square function for the active and reactive branch flows as in [20] or [21]. However, the PWL accuracy is not systematically ensured and this paper shows that great errors can occur when comparing the results with a reference AC OPF. Indeed, the linearization depends on optimization parameters set to represent the upper bounds for the line flows (active and reactive). Those parameters are often deemed constant along the simulated horizons, and equal to the line ratings, which leads to strong losses overestimation when the actual flow does not meet this limit. The method proposed in this paper consists in an iterative LDF with successive updates for the optimization parameters in order to leverage the errors on the loss estimations. The main contributions and results of this paper are:

- An iterative LDF, with its performances assessed in terms of voltage and losses error with a reference AC OPF and compared to references LDF, QDF and SOCP.

- An improvement in a ratio from 5 to 40 compared to the loss estimations with conventional LDF.

- A scalable method compared to conventional SOCP and QDF with up to one month simulation (at 1h time step) with 30 DG units on a 69-bus test system.

- A successful integration of the method in typical DG planning problems with the operation, the sizing and the siting of PV + storage units.

The rest of the paper is organized as follows - Section 3 presents the reference models (LDF, SOCP and QDF) for comparison purposes, with a subsection dedicated to their identified limitations. The iterative LDF is then introduced in Section 4 along with modified formulations for DG planning studies. The method is validated over different test systems and applications in section 5 before conclusions are drawn in Section 6.

\section{Reference Models and Motivations}

All the implemented problems are written using YALMIP toolkit [22] in MATLAB 2018b and solved with CPLEX 12.10.0. on a 4 cores i5-825OU processor with 8 Go of RAM and up to 8 threads in parallel.

\subsection{Classical DistFLow Equations}

The original DistFlow formulation for radial systems is based on the iterative computation of the branch flows and node voltages from the slack bus down to the termination buses of the considered radial networks [15]. In (1) and (2), the classical equations are written with positive and negative contributions of the branch flows that may be directional in the framework of DG planning, due to local generation. Thus, every branch should supply or absorb the active and reactive power balance at all its downstream buses (set $D_{b}(l)$ ), additionally to the losses of all the downstream branches (set $D_{l}(l)$ ) represented by the non-linear terms of (1) and (2). As an example, Fig. 1 illustrates the DistFlow equations from the perspective of line $l_{l}$, and with the loss of all the downstream branches implicitly considered as additional loads at the end buses. The sets of all the downstream lines and buses for each branch capture the radial architecture of the network. The voltage drop along each branch is computed following (3) and considering the flow direction and the losses. As already mentioned, the power flow implemented in this paper is intended for dynamic optimization over a representative period, which explains the introduction of the temporal set $T$. Finally, in the absence of distributed generation (i.e. injection at the slack bus only), the bus power balances $P_{b, t}, Q_{b, t}$ are equal to the loads $P_{b, t}^{L}, Q_{b, t}^{L}$

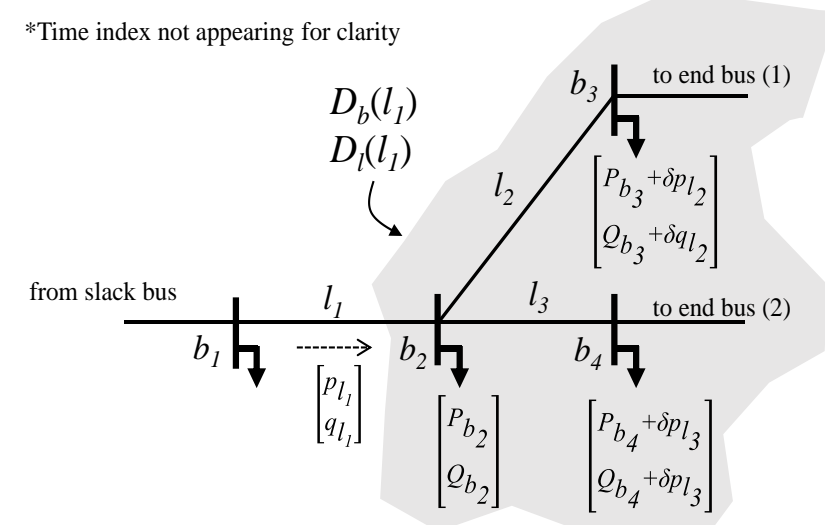

Fig. 1 DistFlow for Radial Networks 


$$
\begin{aligned}
& p_{l, t}=p_{l, t}^{+}-p_{l, t}^{-}=\sum_{b \in D_{b}(l) \subset B} P_{b, t}+\sum_{l \in D(l) \subset L} r_{l} \cdot \frac{p_{l, t}^{2}+q_{l, t}^{2}}{v_{s(l), t}^{2}} \\
&=\sum_{b \in D_{b}(l) \subset B} P_{b, t}+\sum_{l \in D(l) \subset L} \delta p_{l, t} \\
& q_{l, t}=q_{l, t}^{+}-q_{l, t}^{-}=\sum_{b \in D_{b}(l) \subset B} Q_{b, t}+\sum_{l \in D_{l}(l) \subset B} x_{l} \cdot \frac{p_{l, t}^{2}+q_{l, t}^{2}}{v_{s(l), t}^{2}} \\
&=\sum_{b \in D_{b}(l) \subset B} Q_{b, t}+\sum_{l \in D_{l}(l) \subset L} \delta q_{l, t} \\
& v_{e(l), t}^{2}=v_{s(l), t}^{2}-2 \cdot\left(r_{l} \cdot p_{l, t}+x_{l} \cdot q_{l, t}\right)+\left(r_{l}^{2}+x_{l}^{2}\right) \cdot \frac{p_{l, t}^{2}+q_{l, t}^{2}}{v_{s(l), t}^{2}}
\end{aligned}
$$

\subsection{SOCP as a Comparison Model}

One classical approach to solve those equations for scheduling problems (i.e. dynamic optimization for multiperiods OPF) is to perform a convex relaxation of the nonlinear terms in the form of a Second Order Conic Program [13] [23]. Typically, a variable $I_{l, t}$ representing the square of the line current is introduced, along with a variable for the square of the bus voltage $V_{b, t}=v_{b, t}{ }^{2}$. The losses and voltages drops are computed following (4) with the branch impedance. In the conventional SOCP formulation, the current equality constraint is converted into an inequality which is itself rewritten following second order cone constraint [24] ((5)). The relaxation requires that the objective function is convex and monotonically increasing with $I_{l, t}$, which is the case with the objective being the loss minimization as any conventional OPF problem, indiscriminately on the active and reactive components (losses in per unit in (6)). Obvious additional constraints control the current and voltage limits ((7)).

$$
\begin{gathered}
\left\{\begin{array}{l}
\delta p_{l, t}=r_{l} I_{l, t} \\
\delta p_{l, t}=x_{l} I_{l, t}
\end{array} \text { with } I_{l, t}=\frac{p_{l, t}^{2}+q_{l, t}^{2}}{V_{s(l), t}}\right. \\
I_{l, t} \geq \frac{p_{l, t}^{2}+q_{l, t}^{2}}{V_{s(l), t}} \Rightarrow I_{l, t}+V_{s(l), t} \geq\left\|\begin{array}{c}
2 \cdot p_{l, t} \\
2 . q_{l, t} \\
I_{l, t}-V_{s(l), t}
\end{array}\right\|_{2}
\end{gathered}
$$

$o b j: \min \sum_{t \in T} \sum_{l \in L} r_{l} I_{l, t}+x_{l} \cdot I_{l, t}$

$\underline{v_{b}^{2}} \leq V_{b, t} \leq \overline{v_{b}^{2}}$ and $I_{l, t} \leq \overline{i_{l}^{2}}$

\subsection{Quadratic DistFlow as a Comparison Model}

Another formulation considered in this paper as a comparison model consist in a quadratic Disflow (QDF). Similar to the problem formulation considered in [25], the bus voltage is simply set at the reference value for the loss computation in the SOCP equation (4), which then becomes convex and solvable with a quadratic programming solver.

\subsection{Linear DistFlow as a Comparison Models}

Typical linear DistFLow (LDF) equations can consist in neglecting the nonlinear terms that represent the losses as in [17] or [19]. At the same time, the voltage at each bus is assumed to be close to the reference voltage $v_{0}$ with $\left(v_{b, t}-v_{0}\right)^{2} \sim 0$, and (3) is rewritten following (8).

$V_{e(l), t}=V_{s(l), t}-2 \cdot\left(r_{l} \cdot p_{l, t}+x_{l} \cdot q_{l, t}\right)$

However, a quadratic formulation of the losses is sometimes still considered as the objective function of the considered optimization problems. Similar to [17] and [16], the aggregated losses are computed somewhat independently from the grid modelling itself, which does not adequately capture their impact on the voltage drops and line flows (for the upstream lines). More accurate linearized approaches are not new and consist in a piecewise linearization of the square for the active/reactive powers in (4) as in [20] or [21] (Fig. 2). Piecewise linear segments are then introduced for the positive and negative contribution of the branch flows $((9))$, and (10) computes the line square current with the contribution of every PWL block $c$. The slope coefficients as well as lower/upper bounds in each block depend on the maximum line power, with $\overline{p_{l, t}}, \overline{q_{l, t}}$ respectively for the active and reactive flows. Similar to the SOCP, that linearization is valid as long as the approximated function is convex and integrated in the objective. Ultimately the losses are accounted in the voltage drop computation ((11)).

$$
\begin{aligned}
& \left\{\begin{array}{l}
p_{l, t}^{-}=\sum_{c \in C} p_{l, c, t}^{-}, p_{l, t}^{+}=\sum_{c \in C} p_{l, c, t}^{+}, 0 \leq p_{l, c, t}^{-}, p_{l, c, t}^{+} \leq \overline{p_{l}} / C \\
q_{l, t}^{-}=\sum_{c \in C} q_{l, c, t}^{-}, q_{l, t}^{+}=\sum_{c \in C} q_{l, c, t}^{+}, 0 \leq q_{l, c, t}^{-}, q_{l, c, t}^{+} \leq \overline{q_{l}} / C
\end{array}\right. \\
& I_{l, t}=\frac{1}{v_{0}^{2}} \cdot \sum_{c \in C}(2 \cdot c-1) \cdot\left(\frac{\bar{p}_{l} \cdot\left(p_{l, c, t}^{+}+p_{l, c, t}^{-}\right)}{C}+\frac{\overline{q_{l}} \cdot\left(q_{l, c, t}^{+}+q_{l, c, t}^{-}\right)}{C}\right) \\
& V_{e(l), t}=V_{s(l), t}-2 \cdot\left(r_{l} \cdot\left(p_{l, t}+\delta p_{l, t}\right)+x_{l} \cdot\left(q_{l, t}+\delta q_{l, t}\right)\right)
\end{aligned}
$$

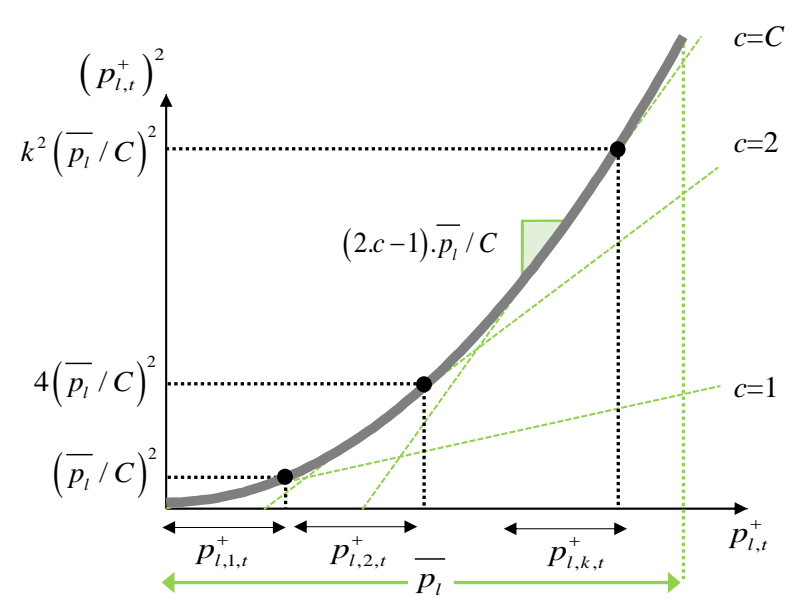

Fig. 2 Conventional piecewise linearization of the square power flow for current/loss computation [20] or [21]

\subsection{Identified Limitations}

Preliminary tests of the reference models are run in this subsection. At first, the time horizon is increased while performing a load flow calculation on the IEEE 69-bus system and with no DG installed (Table 1). Both SOCP and LDF are quickly solved in "snapshot" mode (i.e. a single time step / single load level). 
Table 1 Computational times over different time horizons on the IEEE 69-bus system

\begin{tabular}{c|cccccc}
\cline { 2 - 7 } & snap. & 1day & 2days & 3days & 4days & 7days \\
\hline SOCP & $2 \mathrm{~s}$ & $27 \mathrm{~s}$ & $63 \mathrm{~s}$ & $124 \mathrm{~s}$ & $218 \mathrm{~s}$ & - \\
\hline QDF & $1 \mathrm{~s}$ & $17 \mathrm{~s}$ & $58 \mathrm{~s}$ & $101 \mathrm{~s}$ & $186 \mathrm{~s}$ & - \\
\hline LDF & $<1 \mathrm{~s}$ & $3 \mathrm{~s}$ & $3 \mathrm{~s}$ & $4 \mathrm{~s}$ & $7 \mathrm{~s}$ & $10 \mathrm{~s}$ \\
\hline
\end{tabular}

Table 2 SOCP relaxation inequality value (5) for selected branches (in p.u.) on the IEEE 69-bus system

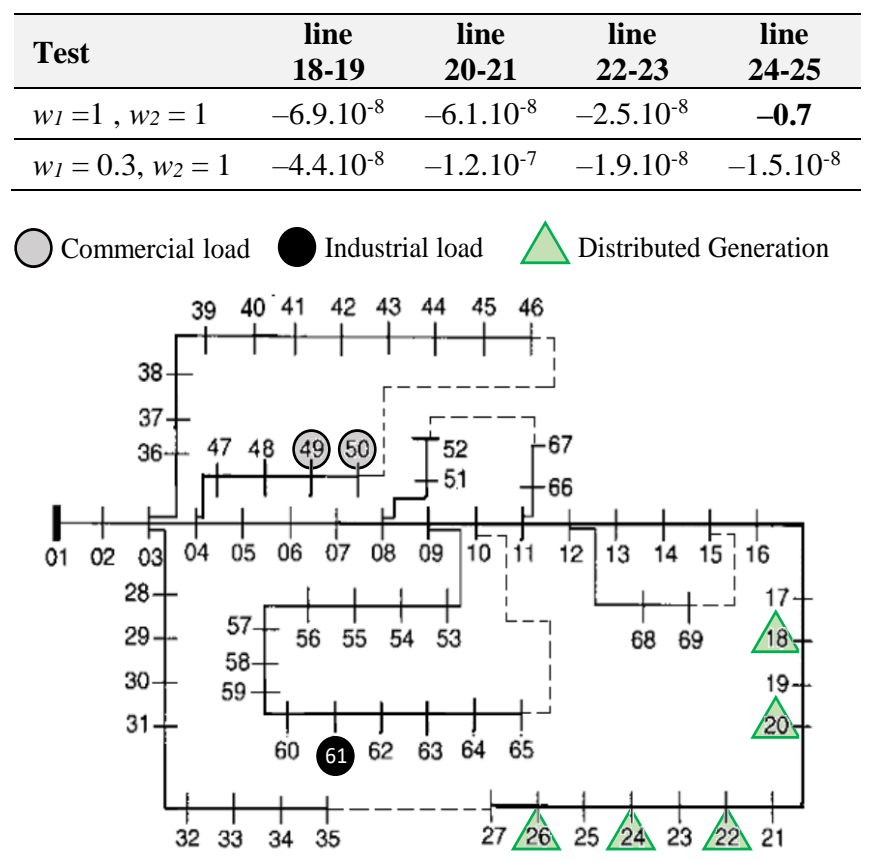

Fig. 3: IEEE 69 bus system with distributed generation

When the temporal set expands, the SOCP computation is significantly greater, with a stack overflow occurring for only one week long simulations - given the computational capabilities and software versions. With strictly linear equations, the LDF is run in some seconds in any case (Table 1). Besides those scalability concerns, the SOCP may suffer from an invalid relaxation of constraint (4). Indeed, as observed in [26], in case of modified objective function such as the maximum usage of local energy resources, the inequality in (5) is not satisfied with an equality. Thus, the obtained solution has no physical meaning despite its mathematical feasibility. Additional preliminary tests then consist in connecting PV systems to the IEEE 69-bus network (arbitrary at buses 18, 20, 22, 24 and 26) (Fig. 3). The SOCP objective is modified in order to integrate the PV curtailment $\Delta p_{b, t}^{p v}$ at every bus (12). Simulations are run over a single time step and while controlling the voltage profiles within the limits. Especially, two tests are performed with different values for the coefficients $w_{1}$ and $w_{2}$, which allow the arbitrage between the losses and the curtailed power. As in [26], with similar weights for the objectives, the obtained solution is physically infeasible (inequality value for line 2425) and appropriate parameter tunning shall then be considered.

$o b j: \min \sum_{t \in T}\left(w_{1} \cdot \sum_{b \in B} \Delta p_{b, t}^{p v}+w_{2} \sum_{l \in L} r_{l} I_{l, t}+x_{l} \cdot I_{l, t}\right)$

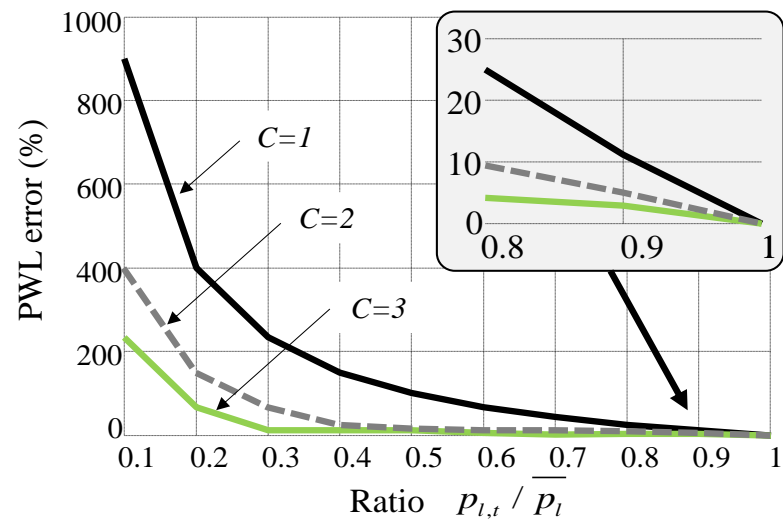

Fig. 4 Overestimation of the PWL formulation for the square function with regard to the ratio actual flow / upper bound

If the conventional LDF allows good scalability for long simulations, one major drawback lies on the piece-wise linearization whose accuracy strongly depends on the chosen values for the upper bounds of the flows $\overline{p_{l, t}}, \overline{q_{l, t}}$. Oftentimes, those values are interpreted as the maximum apparent line power as in [20] or [21] - for both active and reactive power, positive and negative contributions. Therefore, those parameters are deemed constant along the simulated periods. However, the actual flows remain below those limits in most cases (i.e. $p_{l, t} / \overline{p_{l, t}} \leq 1$ for the active power) which may incur strong overestimations in the PWL for the square functions, as depicted in Fig. 4. Those errors naturally tend to decrease with greater numbers of PWL segments but remain important. Fig. 5 displays the actives losses for selected branch, on the IEEE 69-bus system with no DG (i.e. only loads). Especially, the LDF results are compared with a reference AC power flow run in MATPOWER. Following [27], the LDF is performed with two hypotheses in order to set the values for the upper bounds (run over a single time step). At first, both $\overline{p_{l, t}}$ and $\overline{q_{l, t}}$ are the same for all the branches and equal to the summation of the active and reactive loads over all the buses, penalized with a coefficient $(\alpha=1.1)$ that allows to account for the losses (not known a priori). That "conventional" approach denoted as S0 in Fig. 5 considers the same ratings for each line while the branch immediately downstream the slack bus operates close to its limit. In that case, significant deviations (> $200 \%$ for some branches) are observed with much greater estimated losses compared to a reference AC load flow. A second approach individually sets the limit for each branch as the summation of all the loads (active and reactive) located at the downstream buses. With no DG, this method denoted $\mathbf{S} 1$ in Fig. 5 sets $\overline{p_{l, t}}$ and $\overline{q_{l, t}}$ much closer to the actual flow, which leads to a small error in the loss estimation compared to the reference AC load flow.

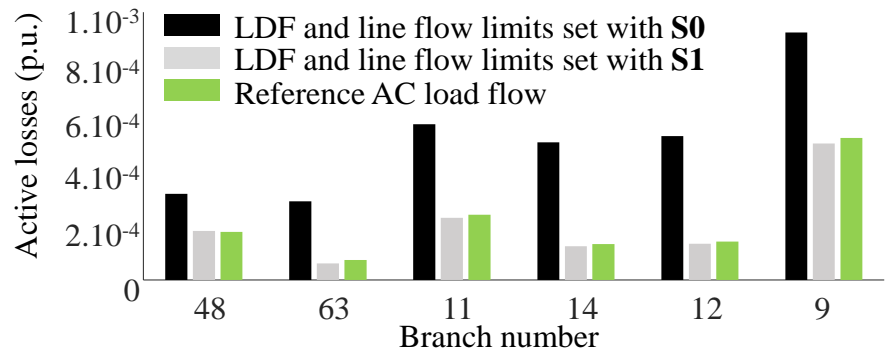

Fig. 5 Branch losses for different settings compared to a reference AC OPF on the IEEE 69 buses system 


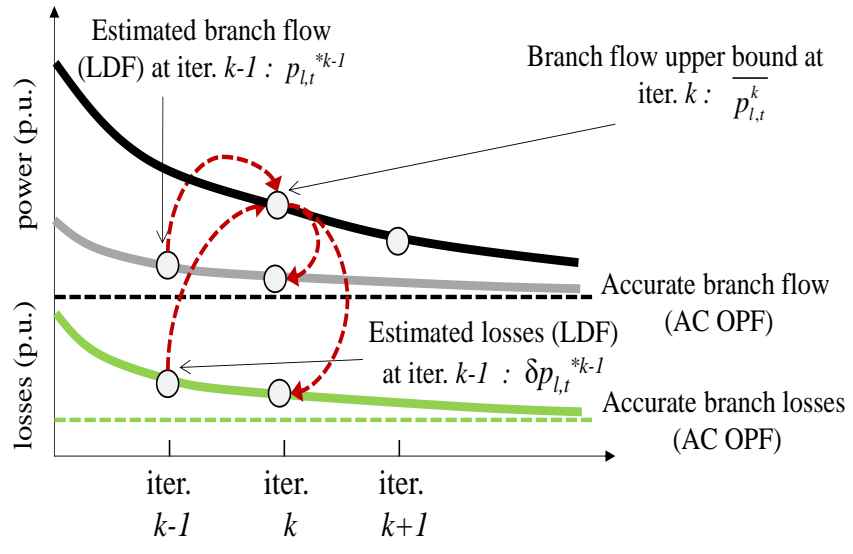

Fig. 6: Proposed successive LDF and optimization parameters update

\section{Proposed Iterative Linear DistFlow for DG planning}

\subsection{Successive $L D F$}

The previous preliminary tests for the LDF showed a higher accuracy when the bounds for the active and reactive flows in the PWL formulation are closer to the actual values. However, when investigating the DG operation (i.e. not only loads), those values cannot be known a priori - i.e it is not possible to guess the line flow values similar to the previous method S1. Especially, the flow distribution depends on the optimal use of the distributed assets which is itself an output of the OPF. This paper proposes an iterative process in order to leverage this shortcoming while successively adjusting the parameter values for the flow bounds in the PWL. Especially, the parameters $\overline{p_{l, t}^{k}}, \overline{q_{l, t}^{k}}$ are introduced with the subscript $k$ denoting an update at every iteration. As illustrated in Fig. 6, this method is expected to refine the losses estimation along with the successive iterations, with decreasing deviations between the flow upper bounds and the estimated values (i.e. the outputs from the LDF at every iteration), which ultimately leads to smaller errors in the PWL and losses computation as previously discussed. In order to further improve the loss estimation, the bus square voltage profiles are similarly estimated at each iteration and injected in the computation of the line current $((10))$. An iterative parameter $V_{b, t}^{k}$ in then introduced and updated at every step. It is important to remind that the parameter values are expected to changes at every time step of the simulated period when dynamic OPF are considered (i.e. time set $T$ ). Ultimately, the PWL constraints of the previous subsection are rewritten following (13) and (14).

$$
\begin{aligned}
& \left\{\begin{array}{l}
p_{l, t}^{-}=\sum_{c \in C} p_{l, c, t}^{-}, p_{l, t}^{+}=\sum_{c \in C} p_{l, c, t}^{+}, 0 \leq p_{l, c, t}^{-}, p_{l, c, t}^{+} \leq \overline{p_{l, t}^{k}} / C \\
q_{l, t}^{-}=\sum_{c \in C} q_{l, c, t}^{-}, q_{l, t}^{+}=\sum_{c \in C} q_{l, c, t}^{+}, 0 \leq q_{l, c, t}^{-}, q_{l, c, t}^{+} \leq \overline{q_{l, t}^{k}} / C
\end{array}\right. \\
& I_{l, t}=\frac{1}{V_{s(l), t}^{k}} \cdot \sum_{c \in C}(2 . c-1) \cdot\left(\frac{\overline{p_{l, t}^{k}} \cdot\left(p_{l, c, t}^{+}+p_{l, c, t}^{-}\right)}{C}+\frac{\overline{q_{l, t}^{k}} \cdot\left(q_{l, c, t}^{+}+q_{l, c, t}^{-}\right)}{C}\right)
\end{aligned}
$$

The implemented procedure then relies on multiple runs of the described LDF - i.e. minimizing the objective (6) subject to constraints (1), (2), (7), (11), (14) and (13). The optimization returns the values for the bus voltages as well as the current, power flows and losses within each line all along the simulated horizon - i.e. $V_{b, t}{ }^{*}, I_{l, t}{ }^{*}, p_{l, t}{ }^{*}, q_{l, t}{ }^{* k}, \delta p_{l, t}{ }^{*}{ }^{* k}, \delta q_{l, t}{ }^{*} k$ with the upperscript ${ }^{* k}$ denoting the LDF optimization output at iteration $k$. The iterative method then updates the values for the bus square voltage at every iteration. For the upper bounds flows, the update is based on optimal branch flows and the losses at all the downstream branches. Similar to the proposition of section 3.4, a penalty coefficient $\alpha$ (typically from in the range [1-1.5]) is introduced in order to ensure the convergence of the linear DistFLow at the next iteration. This coefficient adjusts the bounds for each line flow so that it can supply the losses for the downstream branches. Indeed small upper bounds may endanger the problem feasibility - e.g. in case of DG resources, the optimization at $k+1$ may result in a reverse flow, potentially greater in absolute value than the power computed at iteration $k$. The update process for the corresponding optimization parameters is given in (15) along with the initialization step (i.e. $k=1$ ). At the first iteration, the parameter for the bus voltage used in (13) is then set at the reference value. The upper bounds for the branch are conservatively set to high values corresponding to the summations of the bus loads (both active and reactive) penalized with $\alpha$.

$$
\left\{\begin{array}{l}
\overline{p_{l, t}^{k+1}}=\alpha \cdot\left(\left|p_{l, t}{ }^{* k}\right|+\sum_{l \in D_{l}(l) \subset L} \delta p_{l, t}{ }^{* k}\right) ; \overline{p_{l, t}^{k=1}}=\alpha \cdot \sum_{b \in B} \sum_{t \in T} P_{b, t}^{L} \\
\overline{q_{l, t}^{k+1}}=\alpha \cdot\left(\left|q_{l, t}{ }^{* k}\right|+\sum_{l \in D_{l}(l) \subset L} \delta q_{l, t}{ }^{* k}\right) ; \overline{q_{l, t}^{k=1}}=\alpha \cdot \sum_{b \in B} \sum_{t \in T} Q_{b, t}^{L} \\
V_{b, t}^{k+1}=V_{b, t}{ }^{* k} ; V_{b, t}^{k=1}=v_{0}^{2}
\end{array}\right.
$$

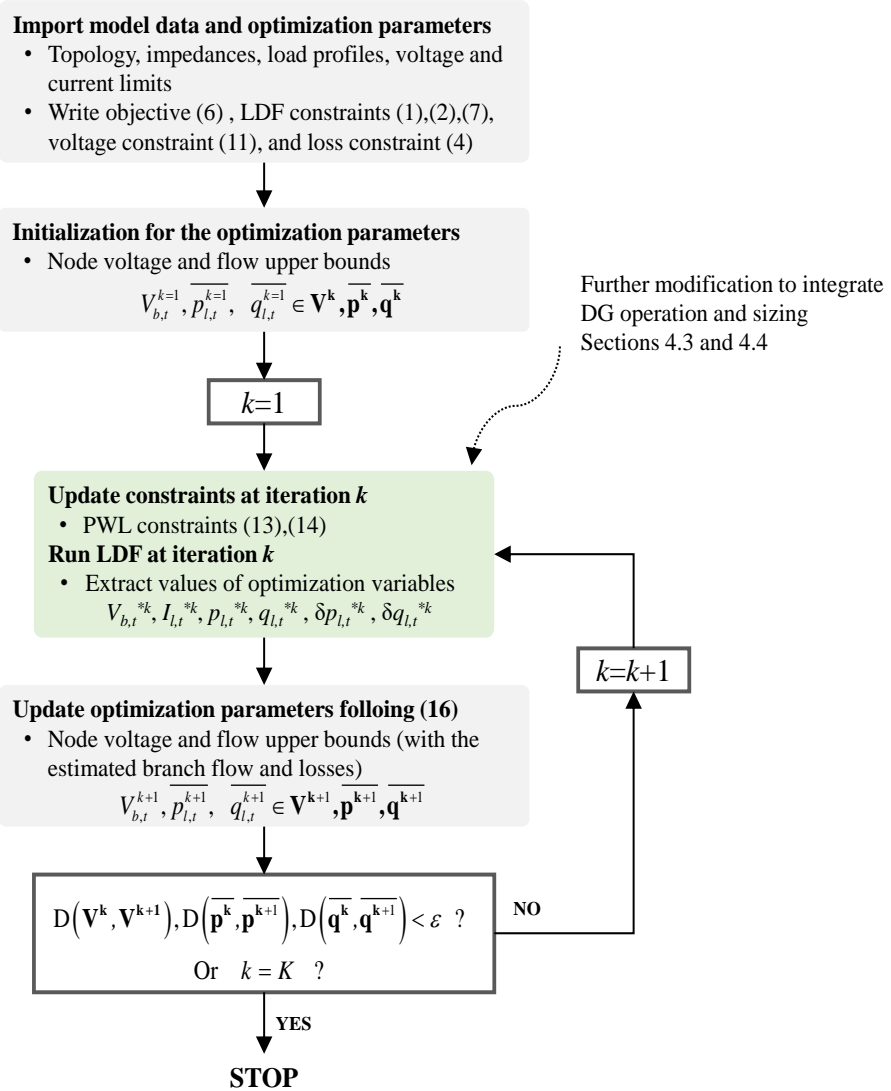

Fig. 7 Proposed algorithm 
The procedure stops whenever a maximum number of iterations $K$ is reached or when the difference between two successive updates for the voltages and flow parameters is below a threshold. That difference is computed as a normalized RMSE between two dimensions parameters $N \times T$ ( $N$ being either the set of lines or buses here) and is noted $\mathrm{D}(\mathbf{X}, \mathbf{Y})((16))$. A threshold at $\varepsilon=1 \%$ is defined, and the iterative procedure stops when, $\mathrm{D}\left(\mathbf{V}^{\mathbf{k}}, \mathbf{V}^{\mathbf{k}}\right), \mathrm{D}\left(\overline{\mathbf{p}^{\mathbf{k}}}, \overline{\mathbf{p}^{\mathbf{k}+\mathbf{1}}}\right)$ and $\mathrm{D}\left(\overline{\mathbf{q}^{\mathbf{k}}}, \overline{\mathbf{q}^{\mathbf{k}+1}}\right)$ are below $\varepsilon$. The proposed procedure is finally summarized in Fig. 7 while detailing the LDF optimization problem successive updates.

$$
\mathrm{D}(\mathbf{X}, \mathbf{Y})=100 \cdot \frac{\sqrt{\frac{1}{N \cdot T} \cdot \sum_{n \in N} \sum_{t \in T}\left(X_{n, t}-Y_{n, t}\right)^{2}}}{\frac{1}{N \cdot T} \cdot \sum_{n \in N} \sum_{t \in T} X_{n, t}}
$$

\subsection{DG Optimal Operation}

The main objective of the proposed approach is to run dynamic optimizations over various periods of time (e.g. day, week, months) and with the integration of DG units. In this section, typical solar generation coupled with storage units are considered. The formulation of the linear DistFlow is modified in order to model the DG operation. The active power balance at each bus is then written as in (17) in order to integrate PV and storage active flows while the reactive balance at each remains unchanged, equals to the reactive consumption. The solar generation is computed following (18) with the rated capacity $P_{b}^{p v R}$ at each bus (if any) and a normalized daily profile $P_{t}^{p v N}$ over the simulated period. Without any operating cost considered (e.g. energy bill, maintenance, etc), the objective to minimize is still the overall system losses $((6))$. Conventional constraints are introduced in order to represent the storage operation and while discriminating the charge and discharge flows penalized by the system efficiency. Typical constraints are related to the maximum charge/discharge flows lower than the rated power $P_{b}^{s t R}$ of the storage at each bus (if any) ((19)). Finally, the state of charge $S O C_{b, t}^{s t}$ should remain within acceptable limits and return to its initial value at the end of the simulated period (i.e. energy conservation with a given rated capacity $\left.E_{b}^{\text {stR }}\right)((20))$. Such an approach could then be integrated in a bi-level optimization framework for optimal siting - i.e. one outer loop investigating different locations for given DG units and an inner loop optimizing their operation (i.e. the iterative modified LDF here) (Fig. 8a).

$$
\begin{aligned}
& \left\{\begin{array}{l}
P_{b, t}=P_{b, t}^{L}-P_{b, t}^{p v}-P_{b, t}^{s t+}+P_{b, t}^{s t-} \\
Q_{b, t}=Q_{b, t}^{L}
\end{array}\right. \\
& P_{b, t}^{p v}=P_{b}^{p v R} \times P_{t}^{p v N} \\
& 0 \leq P_{b, t}^{s t-}, P_{b, t}^{s t+} \leq P_{b}^{s t R} \\
& \left\{\begin{array}{l}
\operatorname{SOC}_{b, t}^{s t}=S O C_{b, t-1}^{s t}-\frac{100 \times \Delta t}{E_{b}^{s t R}} \\
\left.\operatorname{SOC}_{b}^{s t} \leq \operatorname{SOC}_{b, t}^{s t} \leq \frac{S_{b, t}^{s t+} / C_{b}^{s t}}{s t}-P_{b, t}^{s t-} \cdot \eta_{b}^{s t}\right)
\end{array} \operatorname{SOC}_{b, t=T}^{s t}=\operatorname{SOC}_{b, t=1}^{s t}\right.
\end{aligned}
$$

a)

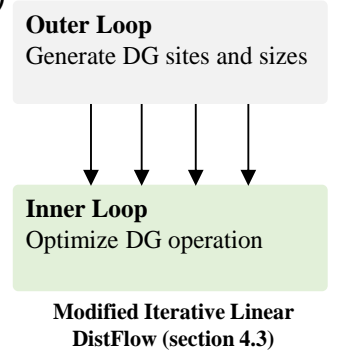

b)

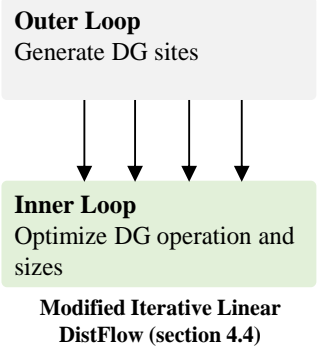

Fig. 8: Optimization frameworks for DG siting/sizing-a) DG optimal operation - b) DG optimal operation/size

\subsection{DG Optimal Operation and Size}

In the previous subsection, the values for the DG rated capacities (i.e. $P_{b}^{p v R}, P_{b}^{s t R}, P_{b}^{p v R}$ ) where considered as known parameters while computing the optimal operation. In the framework of optimal DG sizing studies, those capacities shall be optimized at the same time as the operation and for different potential locations. Thus, this subsection proposes another version of the implemented linear DistFlow with the sizes and controls of DG units as variables of a single optimization problem. The objective function of the iterative LDF is modified in the form of a typical normalized weighted sum [28] in order to account for both system losses and installation costs $((21))$. Especially, the rated power of the storage is sized independently from its capacity which is not commonly done in the literature where a battery capacity reflects its maximum charge/discharge (at $1 \mathrm{C}$-rate). The loss term is normalized with $\delta p_{l, t}^{0}, \delta q_{l, t}^{0}$ (in p.u.) that corresponds to the losses for a base case scenario with no DG. The cost term is normalized with $C_{0}$, as a maximum expected cost with upper bounds for the installed capacities. Finally, the weight $w_{\text {loss }}$ (in $[0,1]$ ) allows an arbitrage between the losses and the installation cost. Indeed, it is important to note that the loss term cannot be removed from the objective function at the expense of losing the convexity of the problem with regard to the linearization process.

$$
o b j: \min \left(\begin{array}{c}
\sum_{l o s s} \cdot \frac{\sum_{t \in T} \sum_{l \in L} \delta p_{l, t}(p \cdot u)+\delta q_{l, t}(p \cdot u)}{\sum_{t \in T} \sum_{l \in L} \delta p_{l, t}^{0}(p \cdot u)+\delta q_{l, t}^{0}(p \cdot u)}+\ldots \\
\ldots .\left(1-w_{\text {loss }}\right) \cdot \frac{\sum_{b \in B} C^{P p \vee R} \cdot P_{b}^{p v R}+C^{P s t R} \cdot P_{b}^{s t R}+C^{E s s t} \cdot E_{b}^{s t R}}{C^{0}}
\end{array}\right)
$$

The operating constraints for the storage are adapted similar to [29] in order to avoid any nonlinearity for the SOC computation. The rated power is limited by the storage capacity with (22). Then the state of charge is implicitly replaced by the amount of energy within the storage while multiplying the SOC constraints by the rated capacity ((23)).

$$
\begin{aligned}
& 0 \leq P_{b, t}^{s t-}, P_{b, t}^{s t+} \leq P_{b}^{s t R} \text { and } \mathrm{P}_{b, t}^{s t-}, P_{b, t}^{s t+} \leq E_{b}^{s t R} / \Delta t \\
& \left\{\begin{array}{l}
S O C_{b, t=0}^{s t} \cdot E_{b}^{s t R}-\sum_{k=1}^{k=t} 100 \times \Delta t \cdot\left(P_{b, k}^{s t+} / \eta_{b}^{s t}-P_{b, k}^{s t-} \cdot \eta_{b}^{s t}\right) \geq \underline{S O C_{b}^{s t} \cdot} \cdot E_{b}^{s t R} \\
S O C_{b, t=1}^{s t} \cdot E_{b}^{s t R}-\sum_{k=1}^{k=t} 100 \times \Delta t \cdot\left(P_{b, k}^{s t+} / \eta_{b}^{s t}-P_{b, k}^{s t-} \cdot \eta_{b}^{s t}\right) \leq \overline{S O C_{b}^{s t}} \cdot E_{b}^{s t R} \\
\sum_{t \in T} 100 \times \Delta t \cdot\left(P_{b, t}^{s t+} / \eta_{b}^{s t}-P_{b, t}^{s t-} \cdot \eta_{b}^{s t}\right)=0
\end{array}\right.
\end{aligned}
$$


In conventional planning problems (e.g. DG allocation, microgrid design), the objective is oftentimes to find a tradeoff between the operating costs and the capital expenditures. In the planning problem considered here there is no energy cost considered (e.g. for the energy imported from the upstream grid). Thus the operating expenses are implicitly represented by a constraint on the self-sufficiency ratio $\alpha^{s s r}$ ((24)) as per defined in [29] and usually computed as the ratio between the energy imported at the Point of Common Coupling (PCC) and the total generation on site. Even though the consideration of that ratio does not capture the losses and is not straightforward in the presence of energy storage ([30]), it allows to capture the degree of autonomy for the considered system.

$$
\sum_{t \in T} P_{t}^{p c c}=\left(1-\alpha^{s s r}\right) \times \sum_{t \in T} \sum_{b \in B} P_{b, t}^{L}
$$

Similar to the simulation of DG operation in the previous section, the modified procedure described here can be integrated in a bi-level optimization framework. An outer loop may investigate different locations and the proposed modified LDF optimizes the DG sizes and operation in each case (Fig. 8b). The outer loop could consist in an analytical method, exhaustive search or metaheuristics and is outside the scope of this paper. A genetic algorithm is considered here.

\section{Validation Runs and Applications}

\subsection{Snapshot and Dynamic Power Flows}

At first, the iterative LDF is validated while running snapshot and dynamic OPF and with no installed DG. Generic IEEE case systems 33-bus, 69-bus and 85-bus are considered as well as a 90-bus distribution network in Cambodia. Table 3 presents the results obtained for different validation runs in a snapshot mode (i.e. single time step) and different numbers of PWL segments $C$. The method performances are considered with regard to the errors compared to the outputs of a reference AC power flow (run in MATPOWER here) and denoted with an upper script ' AC'. Especially the deviations for the voltage and losses are computed using the normalized RMSE in (16) with $\Delta_{V}=\mathrm{D}\left(\left|1-v_{b, t}\right|,\left|1-v_{b, t}{ }^{\mathrm{AC}}\right|\right), \quad \Delta_{\delta P}=\mathrm{D}\left(\delta p_{l, t}, \delta p_{l, t}{ }^{\mathrm{AC}}\right) \quad$ and $\Delta_{\delta Q}=\mathrm{D}\left(\delta q_{l, t}, \delta q_{l, t}^{\mathrm{AC}}\right)$. Note that the error for the voltage considers the deviation with regard to the reference at 1 p.u. Indeed, computing the error in terms of absolute voltage would be more optimistic while dividing by the whole magnitude where deviations between 0.95 p.u. and 1.05 p.u. are usually expected. The iterative LDF is also compared to the results obtained with conventional SOCP, LDF and QDF. For the classical LDF, both approaches S0 and S1 discussed in section 3.4 are considered in order to set the flows upper bounds in the PWL constraints (with 3 PWL blocks here). In snapshot mode, all the simulations for the investigated methods are run in less than 2 seconds. For the iterative modified LDF, significant errors occur with only one or two PWL segments for the loss linearization. However, for $C$ greater or equal than 3 the obtained results are better (both in voltage and losses) than the outputs from conventional SOCP. The QDF is also systematically outperformed with regards to the errors on the losses.
Table 3 Results for snapshot power flows

\begin{tabular}{|c|c|c|c|c|c|c|c|c|}
\hline & \multicolumn{4}{|c|}{ Iterative LDF } & \multirow{2}{*}{ SOCP } & \multirow{2}{*}{$\begin{array}{c}\text { LDF } \\
\text { S0 }\end{array}$} & \multirow{2}{*}{$\begin{array}{c}\text { LDF } \\
\text { S1 }\end{array}$} & \multirow{2}{*}{ QDF } \\
\hline & $C=2$ & $C=3$ & $C=4$ & $C=5$ & & & & \\
\hline \multicolumn{9}{|l|}{ 33-bus } \\
\hline$\Delta_{V}(\%)$ & 1.0 & 0.5 & 0.6 & 0.6 & 0.7 & 3.8 & 0.2 & 0.9 \\
\hline$\Delta_{\delta P}(\%)$ & 10.8 & 1.2 & 1.6 & 1.1 & 2.3 & 46.4 & 7.0 & 8.0 \\
\hline$\Delta_{\delta Q}(\%)$ & 10.7 & 1.3 & 1.5 & 1.1 & 2.4 & 55.9 & 7.9 & 8.8 \\
\hline $\mathrm{Nb}$. iter. & 3 & 3 & 3 & 3 & . & - & - & - \\
\hline \multicolumn{9}{|l|}{ 69-bus } \\
\hline$\Delta_{V}(\%)$ & 1.1 & 0.4 & 0.6 & 0.5 & 0.6 & 2.1 & 0.1 & 1.1 \\
\hline$\Delta_{\delta P}(\%)$ & 10.6 & 1.7 & 2.1 & 1.3 & 2.6 & 26.6 & 9.2 & 10.6 \\
\hline$\Delta_{\delta Q}(\%)$ & 10.9 & 1.6 & 2.2 & 1.2 & 2.3 & 31.2 & 8.0 & 9.3 \\
\hline $\mathrm{Nb}$. iter. & 3 & 3 & 3 & 3 & - & - & - & - \\
\hline \multicolumn{9}{|l|}{ 85-bus } \\
\hline$\Delta_{V}(\%)$ & 1.6 & 0.8 & 1.0 & 0.8 & 1.3 & 4.5 & 0.1 & 1.9 \\
\hline$\Delta_{\delta P}(\%)$ & 9.1 & 2.8 & 2.9 & 2.9 & 4.1 & 36.2 & 11.9 & 13.3 \\
\hline$\Delta_{\delta Q}(\%)$ & 8.9 & 3.0 & 3.0 & 3.1 & 4.3 & 26.6 & 11.2 & 12.7 \\
\hline $\mathrm{Nb}$. iter. & 3 & 3 & 3 & 3 & - & - & - & - \\
\hline \multicolumn{9}{|l|}{ 90-bus } \\
\hline$\Delta_{V}(\%)$ & 0.9 & 0.3 & 0.5 & 0.4 & 0.4 & 2.7 & 0.1 & 0.7 \\
\hline$\Delta_{\delta P}(\%)$ & 12.0 & 0.4 & 2.7 & 0.8 & 1.2 & 60.1 & 5.6 & 6.7 \\
\hline$\Delta_{\delta Q}(\%)$ & 12.0 & 0.4 & 2.7 & 0.8 & 1.2 & 17.4 & 5.5 & 6.6 \\
\hline $\mathrm{Nb}$. iter. & 3 & 3 & 3 & 3 & - & - & - & - \\
\hline
\end{tabular}

Table 4 Iterations Results for Iterative LDF

\begin{tabular}{l|cccc}
\cline { 2 - 5 } & $1^{\text {st }}$ iter & $2^{\text {nd }}$ iter & $3^{\text {rd }}$ iter & $4^{\text {th }}$ iter \\
\hline 33 bus with $\boldsymbol{C}=\mathbf{1}$ & & & & \\
$\Delta_{V}(\%)$ & 18.63 & 4.7 & 3.1 & 3.0 \\
$\Delta_{\delta P}(\%)$ & 284.3 & 99.6 & 62.7 & 59.3 \\
$\Delta_{\delta Q}(\%)$ & 325.6 & 97.1 & 60.9 & 57.9 \\
$\mathbf{3 3}$ bus with $\boldsymbol{C}=\mathbf{3}$ & & & & \\
$\Delta_{V}(\%)$ & 3.9 & 0.7 & 0.5 & - \\
$\Delta_{\delta P}(\%)$ & 46.5 & 1.9 & 1.2 & - \\
$\Delta_{\delta Q}(\%)$ & 55.9 & 1.7 & 1.3 & - \\
\hline
\end{tabular}

As already observed in section 3.4 , the conventional LDF considering the flows bounds as the maximum line power (i.e. LDF S0) displays significant errors in the losses estimation, on average 40 times greater than the iterative approach, which is not acceptable in the framework of DG planning studies. Adjusting those upper bounds closer to the actual flows (LDF S1) reduces those deviations even if the errors remain around five times greater than the values obtained with the proposed procedure. In addition, remind that no DG is considered in those first validation runs which allows to use S1 approach with a confident guess "a priori" for the flow distribution based on the bus loads. It will not be possible when DG are investigated with additional degrees of freedom in the OPF problem that could incur different line flows (e.g. potential reverse flows). The DG operation is itself one expected output of the OPF.

Finally, it is important to note that increasing the number of PWL segments does not necessarily improve the performances of the iterative modified LDF which converge in only three iterations in most of the performed test runs. The successive improvements along the iterations are displayed in Table 4 for the 33 bus systems. Especially, for $C=3$, the errors are greatly reduced right from the second iteration, with a value lower than the results from the traditional SOCP for the same system. 

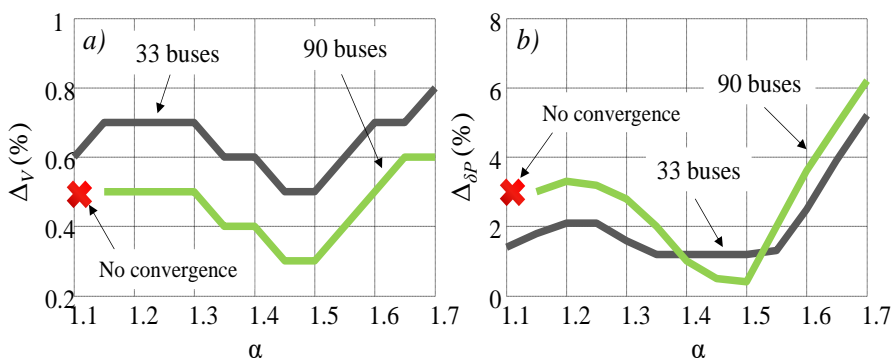

Fig. 9: Impact of $\alpha$ value on voltage and active losses errors for IEEE 33 and 69 buses systems

An important parameter of the proposed iterative LDF is the penalty coefficient $\alpha$ that implicitly accounts for the expected losses within the system. A too small value would incur non convergence (e.g. for the 90 bus system and $\alpha=1.1$ ) as the overall branch flow shall allow to supply the losses. This parameter then needs to be tuned as displayed in Fig. 9 with the errors (in voltage and active losses) computed using different values for $\alpha$ and $C$ fixed to 3 . A value of $\alpha=1.5$ is then set for all the simulations performed in the paper. As a further validation step, Fig. 10 displays the results obtained for the 33 bus system with the voltages and active losses ranked in decreasing order (respectively along with the buses and branches). At the first iteration, the iterative LDF tends to overestimate the losses. The rank of the most lossy branches compared to the reference AC load flow is not even respected (Fig. 10a). Running the iterative procedure allows to almost cancel the errors and to respect the rank of the lossiest branches. The rank of the buses with the lowest voltage is respected from the first iteration (Fig. 10b). A multi-periods load flow is then considered to check that the method can handle dynamic optimization. Fig. 11 displays the accuracy for selected buses and branches while running the iterative LDF on the 69-bus system with a daily load profile. The deviations are very small after three iterations with overall errors below $3 \%$. The accuracy of the iterative $\mathrm{LDF}$ is again better than the SOCP formulation which is run in around 30 seconds ( 3 seconds for the iterative LDF). It is important to remind that this accuracy is allowed by the definition of the PWL parameters all along the time set with no constant value that would correspond to the line ratings.

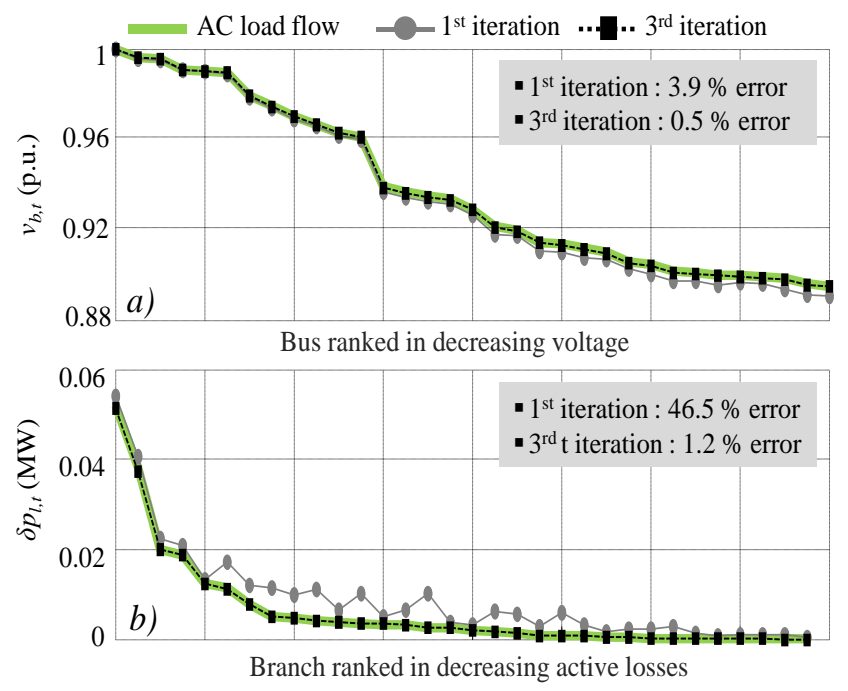

Fig. 10: Results on the 33 bus system - a) bus voltage -b) branch losses
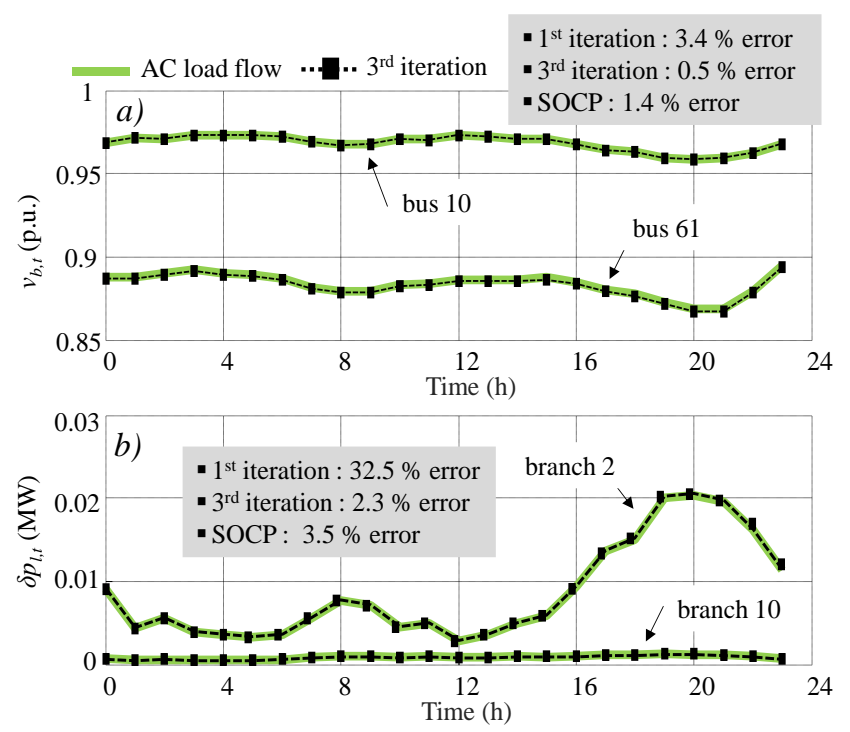

Fig. 11: Daily load flow on the 69 bus system - a) bus voltage -b) branch losses

\subsection{DG Optimal Operation}

Following the previous validation runs and comparisons with reference models, this subsection investigates the use of the proposed iterative LDF for the operation of typical DG units (PV and storage). Daily emulations are run on the IEEE 69-bus populated with time series profiles for residential, commercial (buses 49, 50) and industrial loads (bus 61). Especially, significant DG capacities will be investigated in order to check the physical meaning of the obtained solution, rather than discuss the economical/technical relevance of the use case. A first run of the iterative LDF is performed while installing $300 \mathrm{kWp} \mathrm{PV}$ generators at buses 18, 20, 22, 24 and 26 (Fig. 3). As expected, the obtained results display overvoltage above 1 p.u. at bus 18 (Fig. 12a) with the significant reverse flow in branch 1718 during sun hours (Fig. 12b). 1MW / 1.5MWh storage systems are then added to the PV units at the corresponding bus and the LDF is run while setting a maximum value of 1 p.u. for the buses voltage. In this case the storage are operated so that the voltage at bus 18 remains well below this limit (Fig $12 a$ ) and while minimizing the system losses (i.e. LDF objective function) with limited reverse flow (Fig. 12b). A final simulation is performed with no reverse flow allowed. It can be seen that this binding constraint is well fulfilled (Fig. $12 b)$ with appropriate storage management. Typically, the storage units charge during the sun hours in order to avoid any reverse flow in the upstream grid and discharge at the end of the day in order to further limit the system losses while maximizing local energy usage (Fig. $12 \mathrm{c} \& d$ ). Overall, the feeder downstream bus 18 is operated in an islanded mode with no active import form the upstream network from 8 a.m. onward. Note that following the equations of section 4.2, the DG management only considers the active flows here. More accurate operating areas in the P-Q domain may be defined similar to [17] or [21]. The authors remind that the main objective of the presented applications here is to validate the physical meaning of the obtained results rather that discuss the considered scenarios in terms of installed capacities and/or technical-economic performances. 

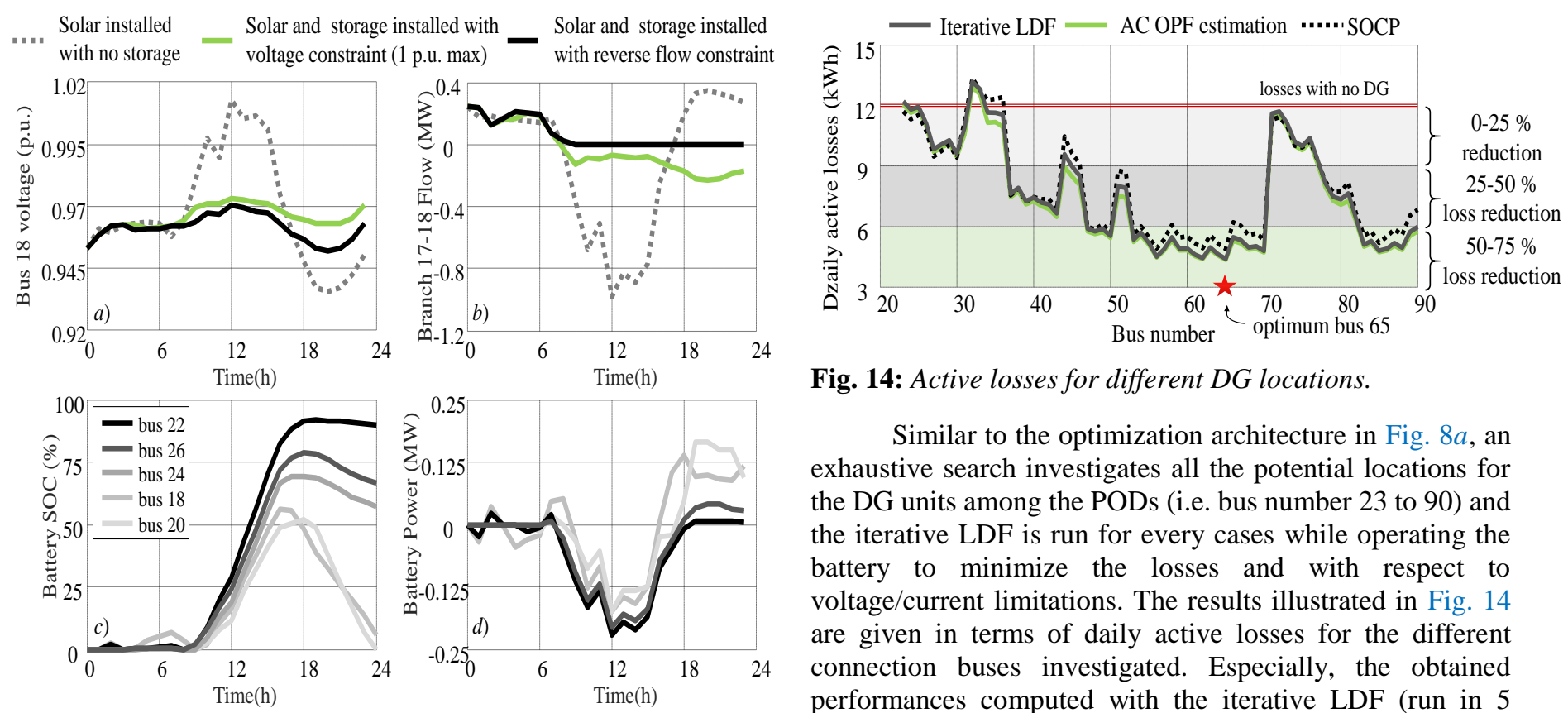

Fig. 12: DG operation on a IEEE 69 buses system - a) bus voltage (18) - b) branch flow (17-18) - c) storage SOC under reverse flow constraint - d) batteries power under reverse flow constraint

\subsection{DG Optimal Siting}

In this subsection, the proposed iterative LDF is integrated in a DG siting problem with the modified equations of Section 4.3. The test system considered is a low voltage $(400 \mathrm{~V})$ 90-bus system in Cambodia whose architecture is displayed in Fig. 13 with a main 150 A feeder and 68 points of delivery (POD) connected through $32 \mathrm{~A}$ lines. The objective is to find the best placement for a PV+Storage unit of given capacities $\left(P_{b}^{p v R}=10 \mathrm{~kW}, P_{b}^{s t R}=10 \mathrm{~kW}\right.$ and $E_{b}^{s t R}=50 \mathrm{kWh}$ ) and while optimizing the operation over a daily simulation. Without any DG (i.e. daily load profiles only) the system displays $12 \mathrm{kWh}$ active losses over the simulated day with severe under voltages around 0.9 p.u.

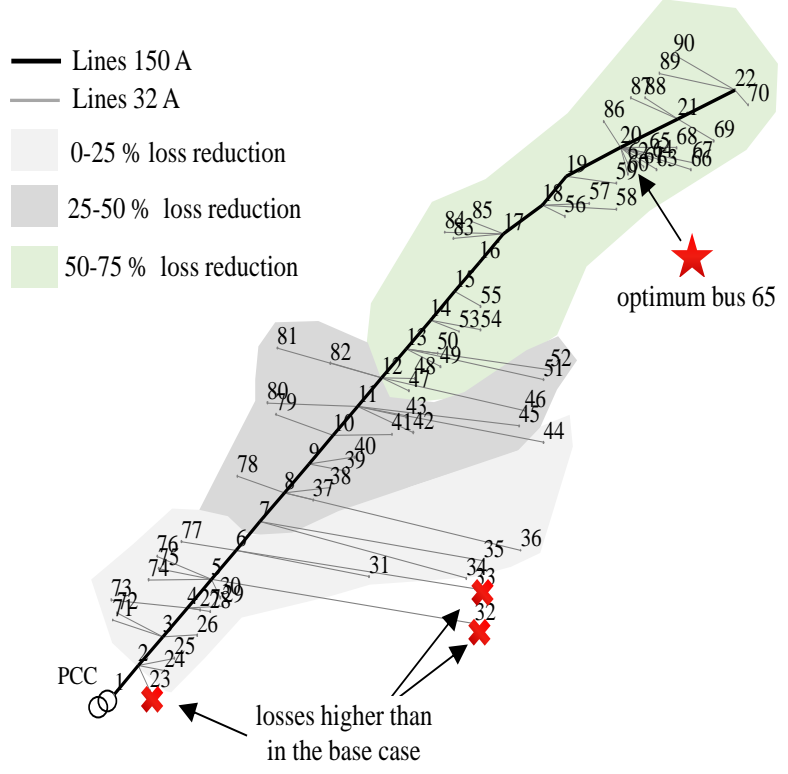

Fig. 13: Considered 90-bus system and optimal siting results

Fig. 14: Active losses for different DG locations.

Similar to the optimization architecture in Fig. $8 a$, an exhaustive search investigates all the potential locations for the DG units among the PODs (i.e. bus number 23 to 90) and the iterative LDF is run for every cases while operating the battery to minimize the losses and with respect to voltage/current limitations. The results illustrated in Fig. 14 are given in terms of daily active losses for the different connection buses investigated. Especially, the obtained performances computed with the iterative LDF (run in 5 minutes) are compared with the outputs from SOCP simulation (run in 1hour to investigate all the nodes). The outcomes are close and with the same rank for the most interesting buses in terms of loss reduction. In addition to its shorter computational time, the iterative LDF displays lower deviations compared to an estimation run with successive AC OPF along the simulated day. Also, the investigation of DG connection at all the nodes allows to partition the PODs in three different clusters in terms of loss reduction; 0-25\%, 25$50 \%$ reduction and $50-75 \%$ reduction. The 'best nodes' are located downstream the main $150 \mathrm{~A}$ line, in an area with a significant amount of load and at PODs located at the end of short 32 A lines (Fig. 13). Also, for the considered system, it is interesting to note that the connection of DG units at some PODs tends to increase the active losses compared to a base case scenario with no distributed resources connected. Typically, those PODs are located close to the PCC and/or at the end of long 32 (high impedance lines).

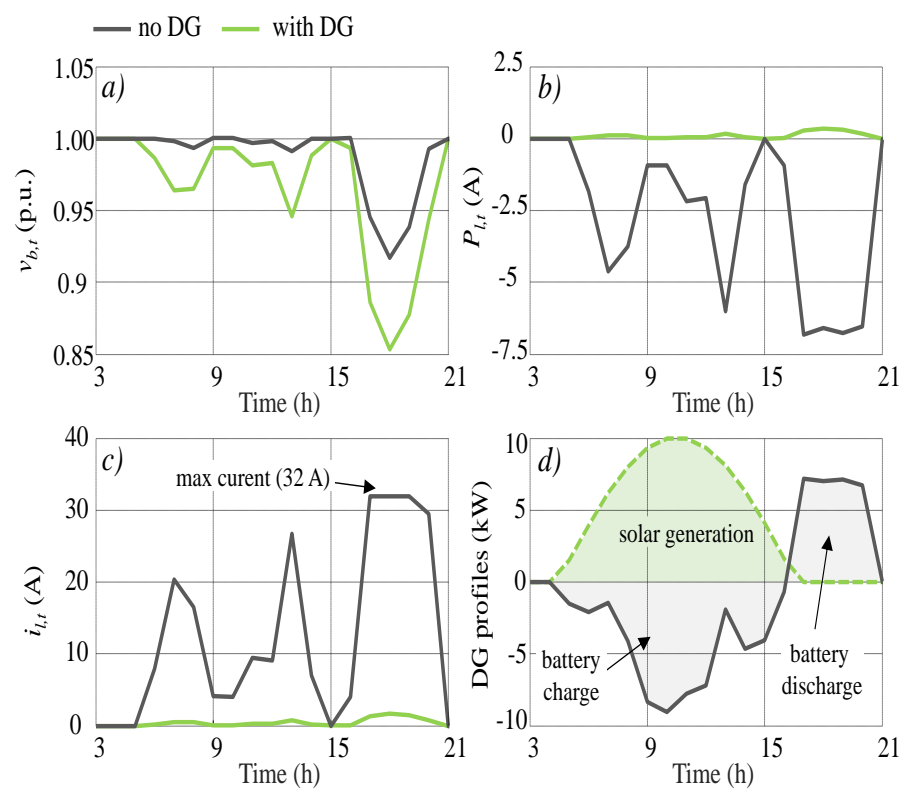

Fig. 15: Results before and after DG installation - a) connection bus voltage - b) upstream line power - c) upstream line current $-d) P V+$ storage operation 
Similar to the results analysis of section 5.2, Fig. 15 displays time series profiles with DG connected at bus 65 in order to validate the physical meaning of the obtained results. With DG capacities greater than individual load profiles, there is a strong reduction of the undervoltage (Fig. 15a) and the corresponding PODs 'exports' energy to the rest of the system (i.e. negative branch flow in Fig. 15b). This export is limited by the 23 A current limit (Fig. 15c), and is allowed by significant installed PV. The PV surplus is stored during the sun hours before the battery is discharged at the end of the day (Fig. 15d). Ultimately the overall system losses are lower while importing less energy from the upstream grid.

\subsection{DG Siting and Sizing Results}

As the last example for the application of the proposed iterative LDF, this subsection investigates the integrated siting, sizing and management of distributed resources. Following the architecture depicted in Fig. 8b, a genetic algorithm generates different sets of potential connection bus(es). In order to lower the combinatorial complexity for the considered 90-bus case, the candidate nodes are the 'best buses'(in terms of loss reduction) in each cluster previously identified. Ten potential buses per cluster are then selected and the genetic algorithm may investigate any combination among those 30 potential buses. In every case, the DG size and operation are optimized according to the modified equations in section 4.4. The objective is to perform an arbitrage between the installation cost and the system losses (parameter $w_{\text {loss }}$ in (21)) and while ensuring a given ratio of self-sufficiency $((24))$. One day simulation is considered.

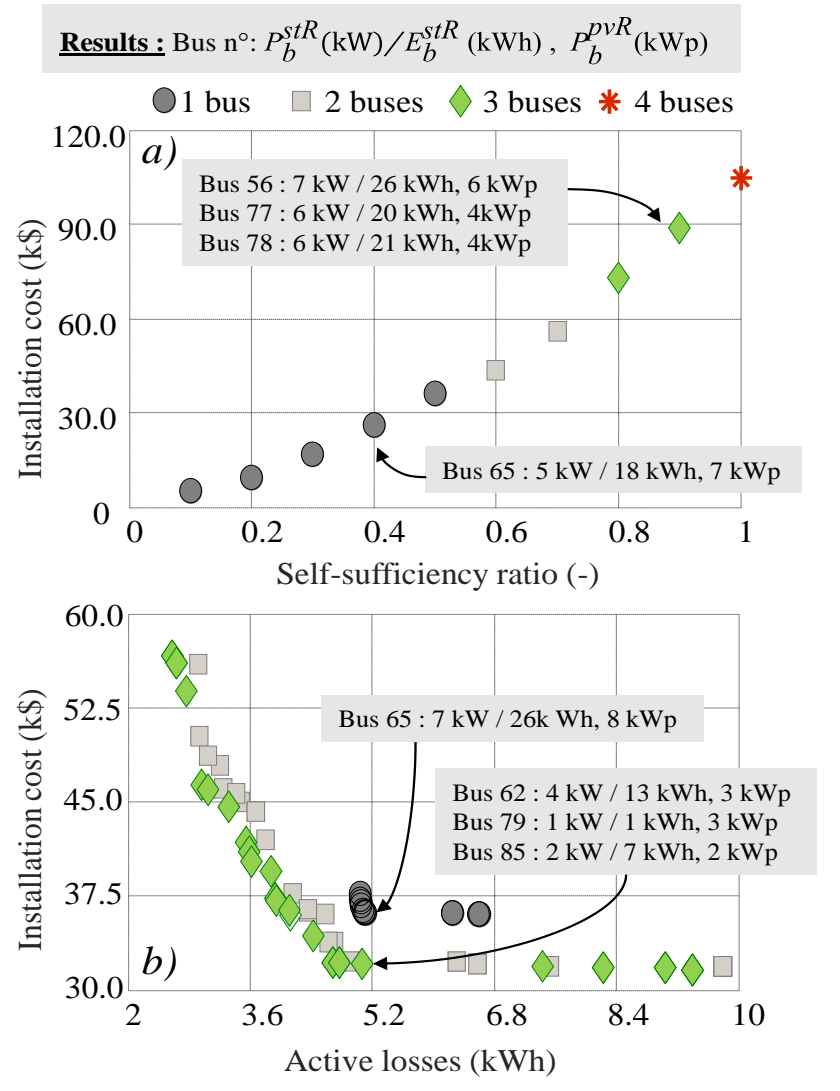

Fig. 16: Siting/Sizing results - a) Optimal cost and connection bus(es) for different self-sufficiency - $b$ ) Cost/losses Pareto fronts for different number of connection buses and $\alpha s s r=0.5$.
The obtained results after several runs with different parameters are given in Fig. 16. Obviously, when greater self sufficiency is required, more DG capacities shall be installed, which incur increasing costs (Fig. 16a). As observed in the previous section, for the considered test system, the maximum line current (32 A), is a binding constraint which limits the size of the DG installed at the POD level. Thus, for higher self-sufficiency $\left(\alpha^{s s}>0.5\right)$ the installed PV and storage capacities shall be distributed among different PODs, up to four different buses necessary for a self-sufficient system (for the considered simulation period). Finally, all the tested configurations in the course of the genetic algorithm allow to investigate the impact of the number of connection buses. Thus for a given value of self-sufficiency (e.g. $\left.\alpha^{s s r}=0.5\right)$, Fig. $16 b$ identifies the best solutions losses/cost (Pareto front), while changing the value of the weight $w_{\text {loss }}$ and the number of connection buses. It is noticeable that distributing the capacities among greater numbers of buses (one to three in the example), allows to significantly reduce the system losses .with shorter distances between the local generation and PODs. Reduced costs are also observable. However, multiplying the number of equipment may lead in the same time to more complex control schemes. There should then be a trade-off between cost and complexity, which is outside the scope of the short study presented in this section.

\subsection{Scalability Tests}

The previous runs performed daily simulations for optimal operation, sizing and siting of the resources. The objective was the integration of the proposed iterative LDF into such problems rather that discuss the obtained performances. Especially, a single day simulation is not enough to represent the systems lifetime (e.g. seasonality effects, aging). Thus, a final set of simulations consists in scalability tests while increasing the length of the simulated period (i.e. the temporal set $T$ ) and the number of connected distributed resources on the IEEE 69-bus test system. The objective is to determine whether the proposed approach can run a longer simulation with the optimal operation of greater numbers of units.

Table 5 Computational time in seconds for different scalability runs of the iterative LDF

\begin{tabular}{c|c|c|c|c|c|c}
\hline \multicolumn{1}{|c|}{ DG $^{\text {Tday }}$} & $\mathbf{1}$ & $\mathbf{7}$ & $\mathbf{1 5}$ & $\mathbf{3 0}$ & $\mathbf{6 0}$ & $\mathbf{9 0}$ \\
\hline $\mathbf{1}$ & 7 & 35 & 93 & 92 & 237 & 590 \\
$\mathbf{1 0}$ & 6 & 46 & 30 & 128 & 273 & - \\
$\mathbf{2 0}$ & 6 & 46 & 40 & 129 & 319 & - \\
$\mathbf{3 0}$ & 8 & 90 & 50 & 396 & - & - \\
$\mathbf{4 0}$ & 9 & 197 & 110 & 425 & - & - \\
$\mathbf{5 0}$ & 13 & 253 & 130 & - & - & - \\
\hline
\end{tabular}

The results displayed in Table 5 show that the iterative LDF allows simulations up to one month at an hourly time step and with more than 30 DG units (PV + Storage) - with a maximum computational time arbitrary set to $600 \mathrm{~s}$. For the same stability test, the SOCP exceeds the $600 \mathrm{~s}$ limit with only three successive days simulated and less than 10 distributed units. With the fully linear problem and the 
available computation capabilities and software versions, the solving time is above the limit for around two million variables. It then defines a 'solvable' area that depends on the size of the system ( $B$ buses) the number of simulated time steps $T$ and the amount of DG units $N$ with $T \times(13 \times B+3 \times N) \leq 2.10^{6}$.

\section{Conclusions}

This paper successfully implements an iterative linear DistFlow for grid modelling purposes in dynamic optimization. The proposed formulation is intended to be used for DG planning studies such as the optimal operation, siting and/or sizing and while considering long time horizons. The iterative LDF is compared with reference models such as LDF, QDF and SOCP while estimating the errors with a reference AC OPF. The iterative LDF displays the best performances with an accuracy on the loss estimation greater in a magnitude of five to forty compared to conventional LDF. When dynamic optimization is considered, the method outperforms the SOCP and QDF with much faster simulations (i.e. scalability results). However, one drawback of the method identified thus far is that the convergence may not be ensured due to the iterative process if binary variables (e.g. genset start/stop) are investigated. This should be part of further application studies. In the present paper, applications such as DG operation, siting and sizing on 69-bus and 90-bus test systems are given as illustrative studies. The iterative LDF is successfully run and coupled with a genetic algorithm to compute the optimal management/size of DG units with different constraints and objectives (i.e. losses, cost and selfsufficiency). In particular, the obtained results show that greater numbers of DG units allow to adjust the trade-off between the cost of installation and system losses. Such problems shall be further investigated and discussed with the proposed method and the inclusion of seasonality, aging and/or uncertainties effects.

\section{References}

[1] R. Jordehi, "Allocation of distributed generation units in electric power systems: a review", Renew. Sustain. Energy Rev., vol. 56, pp. 893-905, Apr. 2016.

[2] P.Paliwal, N.P. Patidar, R.K. Nema, "Planning of grid integrated distributed generators:a review of technology, objectives and techniques", Renew. Sustain. Energy Rev., vol. 40, pp. 557-570, Dec. 2014.

[3] R. Rigo-Mariani, K.V. Ling, J. Maciejowski, "A clusterized energy management with linearized losses in the presence of multiple types of distributed generation”, Int. J. Electr. Power Energy Syst., vol. 113 pp 9-22, May 2019.

[4] H.M. Ayres, D. Salles, W. Freitas, "A practical second-order based method for power losses estimation in distribution systems with distributed generation", IEEE Trans. Power. Syst., vol. 29, no. 2, pp. 666-674, Mar. 2014.

[5] Q. Li, R. Ayyanar, V. Vittal, "Convex optimization for DES planning and operation in radial distribution systems with high penetration of photovoltaic resources", IEEE Trans Sustain Energy, vol. 7, no. 3, pp. 985-995, Jul. 2016.

[6] U.Sultana, A.B.Khairuddin, M.M. Aman, A.S. Mokhtar, N. Zareen, "A review of optimum DG placement based on minimization of power losses and voltage stability enhancement of distribution system", Renew. Sust. Energy. Rev., vol. 63, pp. 363-378, 2016.

[7] R. Arulraj, N. Kumarappan, "Optimal economic-driven planning of multiple DG and capacitor in distribution network considering different compensation coefficients in feeder's failure rate evaluation", Int. J. Eng. Sci. Technol., vol. 22, pp. 67-77, Feb. 2019.

[8] L.M. Belmino, et. al., "Placement and sizing of distributed generation in distribution system", IEEE PES ISGT Latin America, Gramado, Brazil, Nov. 2019.

[9] A.M. Pasha1, H.H. Zeineldin1 E.F. El-Saadany, S.S. Alkaabi, "Optimal allocation of distributed generation for planning master-slave controlled microgrids", IET Gener. Transm. Dis., vol. 13, no. 16, pp. 3704-3712, Aug. 2019.

[10] R.S. Pinto, C. Unsihuay-Vila1, T.S.P. Fernandes, "Multiobjective and multi-period distribution expansion planning considering reliability, distributed generation and self-healing", IET Gener. Transm. Dis., vol. 13, no. 2, pp. 219-228, Dec. 2018.

[11] Y. Zhang, X. Yan, H. Yang, Z.Y. Dong, "Voltage regulationoriented co-planning of distributed generation and battery storage in active distribution networks", vol. 105, pp 79-88, 2019.

[12] M. J. Morshed, J. B. Hmida, A. Fekih, “A probabilistic multiobjective approach for power flow optimization in hybrid wind-PV-PEV systems", Applied Energy, vol. 211, pp. 11361149, 2018.

[13] H. Xing, X. Sun, "Distributed generation locating and sizing in active distribution network considering network reconfiguration", IEEE Access, vol.5, pp. 14768-14774, Jul. 2017.

[14] R. Villasana, L.L. Garve, S.J. Salon, “ Transmission network Using Linear Programming”, IEEE Trans. Power Apparatus and Systems, vol. 104, no. 2, Feb. 2015.

[15] M. E. Baran and F. F.Wu, "Optimal capacitor placement on radial distribution systems," IEEE Trans. Power Del., vol. 4, no. 1, pp. 725-734, Jan. 1989.

[16] H.G. Yeh, D.F. Gayme, S.H. Low, "Adaptive VAR Control for Distribution Circuits With Photovoltaic Generators", IEEE Trans. Power. Syst., vol. 27, no. 3, pp. 1656-1663, Aug. 2012.

[17] K. Turitsyn, P. Sulc, S. Backhaus, M. Chertkov, "Distributed Control of Reactive Power Flow in a Radial Distribution Circuit with High Photovoltaic Penetration", IEEE PES General Meeting, Providence, RI, USA, Jul. 2010.

[18] Z. Wang, B. Chen, J. Wang, J. Kim, "Decentralized Energy Management System for Networked Microgrids in GridConnected and Islanded Modes", IEEE Trans. Smart Grid, vol. 7, no. 2, pp. 1097-1105, Mar. 2016.

[19] Y. Zheng, Y. Song, A. Huang, D.J. Hil, "Hierarchical optimal Allocation of battery energy storage systems for multiple services in distribution systems", IEEE Trans Sustain Energy, Oct. 2019.

[20] P. L. Cavalcante, J. Camilo López, J. F. Franco, M. J. Rider, A.V. Garcia, M.R.R. Malveira, L.L. Martins, L. C. M. Direito, "Centralized Self-Healing Scheme for Electrical Distribution Systems", IEEE Trans. Smart Grid, vol. 7, no. 1, pp. 145-155, Aug. 2015.

[21] A.C. Rueda-Medina, J.F. Franco, M.J. Rider, A. PadilhaFeltrin, R. Romero, "A mixed-integer linear programming approach for optimal type, size and allocation of distributed 
generation in radial distribution systems", Electr. Power Syst. Res., vol. 97, pp. 133-143, 2013.

[22] J. Lofberg, "YALMIP: a toolbox for modelling and optimization in MATLAB", IEEE International Symposium on Computer Aided Control Systems Design, New-Orleans, USA,, pp. 282-289, Sep. 2004.

[23] X. Yunyun, C. Xi, W. Qiuwei, Z. Qian, "Second-order conic programming model for load restoration considering uncertainty of load increment based on information gap decision theory", Int. J. Electr. Power Energy Syst., vol. 105, pp 151-158, 2019.

[24] M. Farivar, S. H. Low, "Branch Flow Model: Relaxations and Convexification-Part I", IEEE Trans Power Syst, vol 28, no. 3, pp 2554-2564, 2013.

[25] D. A. Quijano, A. Padilha-Feltrin, "Optimal integration of distributed generation and conservation voltage reduction in active distribution networks", Int. J. Electr. Power Energy Syst., vol. 113, pp 197-207, May 2019.

[26] K. Christakoua, D.C. Tomozeic, J.Y Le Boudec, M. Paolone, "AC OPF in radial distribution networks - Part I: On the limits of the branch flow convexification and the alternating direction method of multipliers", Electr. Power Syst. Res., vol. 143, pp. 438-450, 2017.

[27] R. Rigo-Mariani, V. Debusshere, M.C. Alvarez-Herault, “A Modified DistFlow for Distributed Generation Planning Problems in Radial Grids", 46th Annual Conference of the IEEE Industrial Electronics Society (IES), IECON, Singapore (Online), 2020.

[28] R.T. Marler ,J.S. Arora, "The weighted sum method for multiobjective optimization: new insights", Struct. Multidisc. Optim., Dec. 2009.

[29] H. Pandžić, Y. Wang, T. Qiu, Y. Dvorkin, D.S. Kirschen, "Near-optimal method for siting and sizing of distributed storage in a transmission network", IEEE Trans Power Syst, vol. 25, no. 1, pp. 2288-3000, 2015 .

[30] P. Balcombea, D. Rigbyc, A. Azapagic, "Energy selfsufficiency, grid demand variability and consumer costs:Integrating solar PV, Stirling engine CHP and battery storage”, Appl. Energy, vol. 155, pp. 393-408, Oct. 2015. 